\title{
Kimura Disease: A Rare Case Report
}

\author{
Rahul Suryakant Abhange*, Rahul Pundalik Jadhav and Neeraj Kumar Jain \\ Department Of Pathology, Government Medical College, Latur, India
}

\begin{abstract}
Kimura disease is a benign rare chronic inflammatory disorder of unknown etiology that involves the lymph nodes and subcutaneous tissue of the head and neck region. Most cases have been described predominantly in middle aged Asian people. The lesion is benign, but it may easily be mistaken for a malignant tumor. Kimura disease has been confused with angiolymphoid hyperplasia with eosinophilia (ALHE), from which it probably should be distinguished as a separate entity. The diagnosis may be suggested by a fine needle aspirate, but is established by a biopsy. The treatment of choice is surgical removal. We hereby, present a case of Kimura disease in 18 year old male presented with multiple swellings over bilateral retro-auricular and occipital region.
\end{abstract}

Keywords: Kimura Disease, Eosinophilia.

\section{Introduction}

Kimura disease is a rare, chronic inflammatory disorder of unknown cause, primarily seen in young Asian males. [1,2] The typical clinical presentation is characterized by a triad of painless unilateral cervical adenopathy or subcutaneous masses predominantly in the head or neck region, blood and tissue eosinophilia, and markedly elevated serum immunoglobulin E (Ig E) levels. ${ }^{[3]}$ Early diagnosis of Kimura's disease may spare the patient from potentially harmful and unnecessary invasive diagnostic procedures.

\section{Case Report}

Our patient, an 18 year-old Indian male, presented with multiple subcutaneous nodules over bilateral retro-auricular and occipital region since two years. Initially, swelling appeared in the occipital region. This swelling grew slowly and was not associated with pain or local skin changes. He also noticed similar but smaller nodules behind the left and right retro-auricular region. His medical, surgical, and family histories were noncontributory. He was not taking medications, and he had no drug allergies.

Physical findings included multiple lesions bilaterally over retro-auricular and occipital region, largest over occipital region approximately measuring $4 \times 3 \mathrm{~cm}$, soft, non-tender and smooth with no skin changes. There were several similar nodules of various sizes in the right and left retro-auricular region [figure 1]. There was no enlarged thyroid gland or axillary adenopathy, and there were no ear or pharyngeal lesions. Similarly, there was no hepatosplenomegaly. A fine needle aspiration was done which revealed lymphoid population of cells along with numerous eosinophils, a diagnosis of Kimura disease was suspected.
An Excisional biopsy of occipital nodule was done, which, on gross examination appeared grey white on external and cut surface and measured $3.5 \times 3 \times 1 \mathrm{~cm}$. Microscopic examination revealed a lymph node showing fibrous capsule with preserved architecture along with follicular hyperplasia with prominent germinal centers [Figure 2]. Germinal centers were vascularized and contained polykaryocytes. There was perifollicular, para-cortical infiltration of mature eosinophils, [Figure 3] along with few plasma cells. Post-capillary venular hyperplasia also noted.

Considering the classic histological and clinical findings a diagnosis of Kimura disease was made.

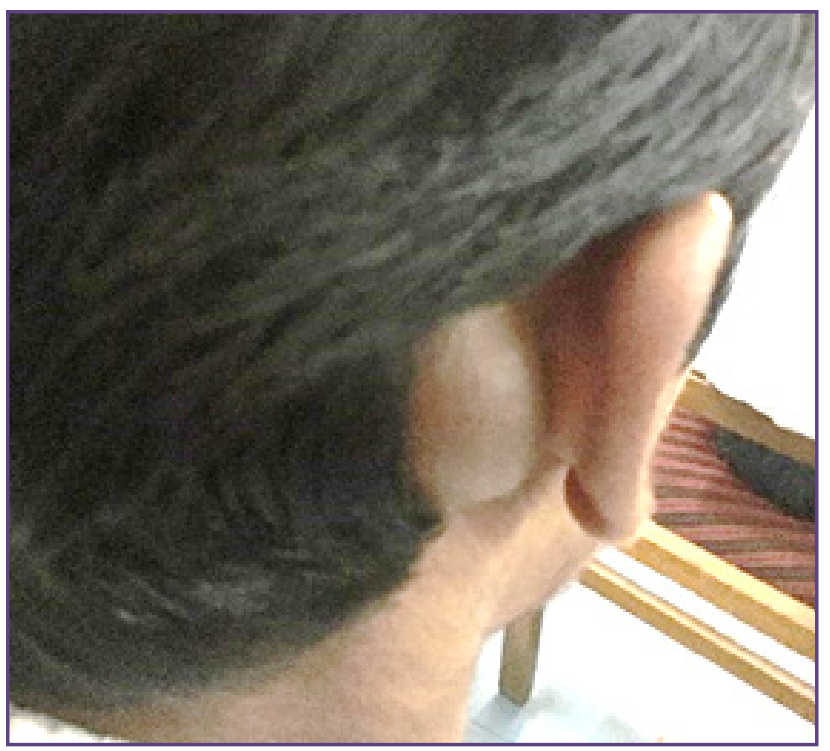

Fig. 1: Soft, non-tender and smooth nodule behind right ear with no skin changes. 


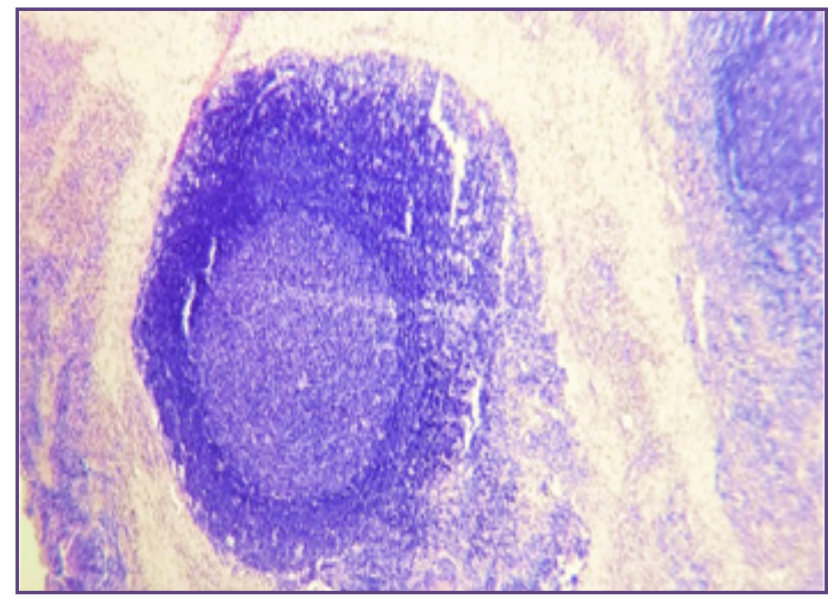

Fig. 2: Follicular hyperplasia with prominent germinal centers. (H \& E. 10x).

\section{Discussion}

Kimura disease was first described in China in 1937, but it was not referred to as Kimura disease until its description in the Japanese language literature in $1948 .{ }^{[4,5]}$ The etiology of this rare disease remains unknown, but it is thought to be an immune-mediated disorder and not neoplastic. The disease is endemic in Asia and sporadic elsewhere; has a male predominance, and presents with painless soft tissue nodules and lymphadenopathy in the head and neck region. Common sites of involvement are the parotid glands and the epi-trochlear region, axillary, and inguinal nodes. Although the masses enlarge slowly, patients remain otherwise asymptomatic. Pruritus and dermatitis may occur, and skin lesions can present as reddish brown papules or as subcutaneous nodules. Rare sites of involvement include the kidneys, orbits, ears, spermatic cord, and nerves ${ }^{[5]}$ Laboratory findings include peripheral eosinophilia and elevated serum Immunoglobulin E levels, and the diagnosis is made histologically, preferably from a lymph node biopsy. Histologically Kimura disease presents as preserved lymph node architecture with reactive and prominent germinal centers. Dense eosinophilic infiltration of the inter-follicular zones, lysis of the follicles, and occasionally microabscesses are seen. Granuloma formations contain infiltration of eosinophils, lymphocytes, plasma cells, and histiocytes. Tissue fibrosis, sclerosis, and vascular proliferation are also present. Vessels remain thin-walled with cubical endothelial cells present. Rarely the features include progressive destruction of germinal centers, presence of polykaryocytes (which are not pathognomic for that disease). Immunofluorescence tests show germinal centers containing heavy Ig E deposits and variable amounts of Ig $\mathrm{G}$, Ig M, and fibrinogen..$^{[6-8]}$

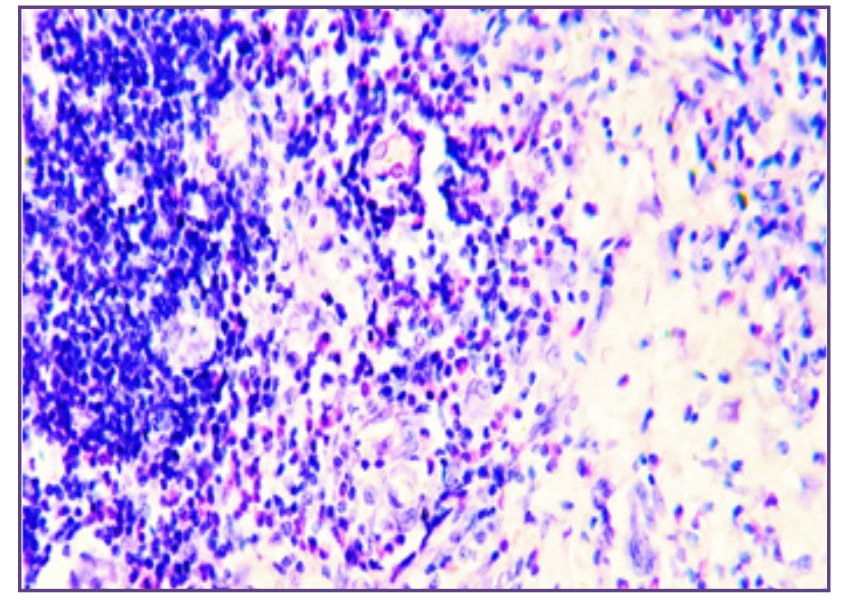

Fig. 3: Eosinophilic infiltration into the inter-follicular zones. (H \& E. 40x).

The treatment of Kimura disease has mainly involved the use of oral corticosteroids. Radiation treatment is usually used for the local control of lesions not responsive to steroids. Irradiation should be considered not only in patients resistant to steroids, but also in young patients in whom the longterm side effects of steroids may be more deleterious than a limited course of irradiation that may prevent relapse. ${ }^{[9]}$ Other treatments have included complete surgical excision, the intra-lesional administration of steroids, cytotoxic agents, and electrodessication. Although spontaneous resolution has been reported, most patients have a prolonged course with slow enlargement of the masses. There is no potential that the lesions will become malignant.

\section{Conclusion}

Kimura disease is a rare benign chronic inflammatory disorder of unknown etiology and pathogenesis. Histopathological examination is most important in its diagnosis. Kimura disease should be kept in mind whenever we come across a middle aged Asian male patient presenting with multiple painless swellings in head and neck region.

\section{Reference:}

1. Kimura T, Yoshimura S, Ishikaura E. Unusual granulation combined with hyperplastic changes of lymphatic tissue. Trans Soc Pathol Jpn. 1948;37:179-180.

2. Kim BH, Sithian N, Cucolo GF. Subcutaneous angiolymphoid hyperplasia (Kimura disease). Report of a case. Arch Surg.1975;110 :1246- 1248.

3. Armstrong WB, Allison G, Pena F, Kim JK. Kimura's disease: two case reports and a literature review. Ann Otol Rhinol Laryngol.1998;107:1066-1071.

4. Irish JC, Kain K, Keystone JS, Gullane PJ, Dardick I. Kimura's disease: an unusual case of head and neck masses. J Otolaryngol. 1994 Apr;23:88-91. 
5. Lee YS, Ang HK, Ooi LL, Wong CY. Kimura's disease involving the median nerve: a case report. Ann Acad Med Singapore 1995;24(3):462-464.

6. Li TJ, Chen XM, Wang SZ, Fan MW, Semba I, Kitano M. "Kimura's disease. A clinicopathologic study of 54 Chinese patients". Oral Surgery, Oral Medicine, Oral Pathology. 1996;82(5):549-555.

7. Ranka SR, Rajput A, Kantharia CV. "Kimura's disease".
Indian Journal of Otolaryngology and Head and Neck Surgery. 2004;56(1):43-45.

8. Armstrong WB, Allison G, Pena,F, Kim JK."Kimura's disease: two case reports and a literature review". Annals of Otology, Rhinology and Laryngology. 1998;107(12):1066-1071.

9. Itami J, Arimizu N, Miyoshi T, Ogata H, Miura K. Radiation therapy in Kimura's disease. Acta Oncol. 1989;28(4):511-514.

*Corresponding author:

Dr. Rahul Suryakant Abhange, Associate Professor,Department Of Pathology, Government Medical College, Latur, 413512, India

Phone: +919561871746

Email: abhangerahul24@gmail.com

Financial or other Competing Interests: None. 